\title{
Molecular Mechanisms and Function Prediction of Long Noncoding RNA
}

\author{
Handong Ma, Yun Hao, Xinran Dong, Qingtian Gong, Jingqi Chen, \\ Jifeng Zhang, and Weidong Tian \\ Institute of Biostatistics, School of Life Science, Fudan University, 220 Handan Road, Shanghai 2004333, China \\ Correspondence should be addressed to Weidong Tian, weidong.tian@fudan.edu.cn
}

Received 30 October 2012; Accepted 21 November 2012

Academic Editors: G. P. Chrousos and T. Kino

Copyright (C) 2012 Handong Ma et al. This is an open access article distributed under the Creative Commons Attribution License, which permits unrestricted use, distribution, and reproduction in any medium, provided the original work is properly cited.

\begin{abstract}
The central dogma of gene expression considers RNA as the carrier of genetic information from DNA to protein. However, it has become more and more clear that RNA plays more important roles than simply being the information carrier. Recently, whole genome transcriptomic analyses have identified large numbers of dynamically expressed long noncoding RNAs (lncRNAs), many of which are involved in a variety of biological functions. Even so, the functions and molecular mechanisms of most lncRNAs still remain elusive. Therefore, it is necessary to develop computational methods to predict the function of lncRNAs in order to accelerate the study of IncRNAs. Here, we review the recent progress in the identification of lncRNAs, the molecular functions and mechanisms of lncRNAs, and the computational methods for predicting the function of lncRNAs.
\end{abstract}

\section{Introduction}

Proteins and related protein-coding genes have been the main subject of biological studies for years. However, with the development of RNA sequencing technology and computational methods for assembling the transcriptome, it has become clear that besides protein-coding genes much of the mammalian genome is transcribed, and many noncoding RNA (ncRNA) transcripts tend to play important roles in a variety of biological processes. Understanding the function of ncRNAs has become one of the most important goals of modern biological studies [1-3]. ncRNAs can be classified into several distinct subclasses, including processed small RNAs [4], promoter-associated RNAs [5], and functional long noncoding RNAs (lncRNAs) [6]. The term of lncRNA was introduced to distinguish the special class of ncRNA from well-known small regulatory RNAs (i.e. miRNAs and siRNAs). lncRNAs are generally longer than 200 nucleotides $[3,7,8]$. Recent studies have shown that lncRNAs may act as important cis- or trans-regulators in various biological processes. Mutations in lncRNAs are related with a wide range of diseases, especially cancers and neurodegenerative diseases. Even so, the functions and molecular mechanisms of most lncRNAs are unknown. Though several computational methods have been developed to predict the functions of lncRNAs, it still remains a challenging task, partly owing to the lack of conservation in both the sequence and secondary structures of lncRNAs [9-11]. In this paper, we will summarize the recent progresses and challenges in the identification, molecular mechanism, and function prediction of lncRNAs.

\section{Definition and Classification of lncRNA}

The definition of IncRNA is based on two criteria, the size and the lack of protein-coding potential. In this paper, lncRNA refers to nonprotein-coding RNA longer than $200 \mathrm{nt}$ $[7,10-12]$, which distinguishes it them from mRNA and small regulatory RNA in a relatively satisfying way $[11,13]$. Depending on their relationships with the nearest proteincoding genes, lncRNAs can be classified in three different ways $[12,14,15]$ : (1) sense or antisense: lncRNAs that are located on the same strand or the opposite strand of the nearest protein-coding genes [16]; (2) divergent or convergent: lncRNAs that are transcribed in the divergent or convergent orientation compared to that of the nearest protein-coding genes [12]; (3) intronic or intergenic: lncRNAs that locate 
inside the introns of a protein-coding gene, or in the interval regions between two protein-coding genes $[12,17]$.

\section{Identification of lncRNA}

To identify lncRNAs, the first step is to obtain all transcripts including ncRNAs and mRNAs in cells, and then to distinguish lncRNAs from mRNAs and other types of ncRNAs. Traditional technologies, such as microarray, focus on the identification of protein-coding RNA transcripts. New technologies, such as RNA-Seq, are not limited to the identification of protein-coding RNA transcripts, and have led to the discovery of many novel ncRNA transcripts. The discrimination between lncRNAs and other small regulatory ncRNAs depends on their length. However, the length information alone is not enough to separate lncRNAs from mRNAs, and other criteria are needed for this purpose. Below, we will first briefly introduce new technologies in identifying RNA transcripts, especially ncRNA transcripts. Then, we will review current methods to distinguish lncRNAs from mRNAs.

\subsection{Experimental Methods in Identifying $\operatorname{lncRNA}$}

Microarray. Traditional microarray technologies use predefined probes to determine the expression level of mRNA transcripts and are not appropriate to identify lncRNAs. However, it has been found that a few previously defined mRNAs or some probe sequences actually are lncRNAs; thus, former microarray datasets can be reannotated to study the expression of lncRNAs [60]. With more and more lncRNAs discovered, new probes specific for lncRNAs can be designed. For example, Babak et al. designed probes from conserved intergenic and intragenic region to identify potential ncRNA transcripts [61]. However, microarray is not sensitive enough to detect RNA transcripts with low-expression level. Thus the use of microarray to identify lncRNAs is limited due to the low expression level of many lncRNAs.

SAGE and EST. SAGE (serial analysis of gene expression) technology produces large numbers of short sequence tags and is capable of identifying both known and unknown transcripts. SAGE has been used and proved to be an efficient approach in studying lncRNAs. For example, Gibb et al. compiled 272 human SAGE libraries. By passing over 24 million tags they were able to generate lncRNA expression profiles in human normal and cancer tissues [62]. Lee et al. also used SAGE to identify potential lncRNA candidates in male germ cell [63]. However, SAGE is much more expensive than microarray, therefore is not widely employed in large-scale studies. EST (expressed sequence tag) is a short subsequence of cDNA, and is generated from oneshot sequencing of cDNA clone. The public database now contains over 72.6 million EST (GeneBank 2011), making it possible to discover novel transcripts. For example, Furuno et al. clustered EST to find functional and novel lncRNAs in mammalian [64]. Huang et al. used the public bovinespecific EST database to reconstruct transcript assemblies, and find transcripts in intergenic regions that are likely putative lncRNAs [65].
RNA-Seq. With the development of next generation sequencing (NGS) technologies, RNA-Seq (also named whole transcriptome shotgun sequencing) has been widely used for novel transcripts discovery and gene expression analysis. Compared to traditional microarray technology, RNA-Seq has many advantages in studying gene expression. It is more sensitive in detecting less-abundant transcripts, and identifying novel alternative splicing isoforms and novel ncRNA transcripts. The basic workflow for lncRNA identification using RNA-Seq is shown in Figure 1. RNA-Seq is currently the most widely used technology in identifying lncRNAs. For example, Li et al. applied RNA-Seq to identify lncRNAs during chicken muscle development [66]. Nam and Bartel integrated RNA-Seq, poly (A)-site, and ribosome mapping information to obtain lncRNAs in C. elegans [16]. Pauli et al. performed RNA-Seq experiments at eight stages during zebrafish early development, and identified 1133 noncoding multiexonic transcripts [67]. Prensner et al. used RNA-Seq to study lncRNA in human prostate cancer from 102 prostate tissues and cell lines, and concluded that lncRNAs may be used for cancer subtype classification [68].

RNA-IP. RNA-IP (RNA-immunoprecipitation) is a new method developed to identify lncRNA that interacts with specific protein. Antibodies of the protein are first used to isolate lncRNA-protein complexes. Then, cDNA library is constructed followed by deep sequencing of interacting lncRNAs. Using RNA-IP, Zhao et al. discovered a 1.6-kb lncRNA within Xist that interacts with PRC2 [69].

Chromatin Signature-Based Approach. The above-mentioned methods target on RNA transcripts directly. In contrast, chromatin signature-based approach uses chromatin signatures, such as $\mathrm{H} 3 \mathrm{~K} 4 \mathrm{me} 3$ (the marker of active promoters) and $\mathrm{H} 3 \mathrm{~K} 36 \mathrm{me} 3$ (the marker of transcribed region), to study actively transcribed genes including lncRNAs. In this approach, ChIP-Seq is used to generate genome-wide profiles of chromatin signatures [70], and the transcribed regions are mapped in the genome, where lncRNAs are determined and studied. For example, Guttman et al. identified 1,600 large multiexonic lncRNAs that are regulated by key transcription factors such as p53 and NFkB [71]. The advantage of this approach is its directness in investigating the mechanisms that regulate lncRNA expression.

\subsection{Computational Methods in Identifying $\operatorname{lncRNA}$}

ORF Length Strategy. Unlike protein-coding genes, the start codons and termination codons in lncRNAs tend to distribute randomly. As a result, the ORF length of lncRNAs can hardly extend to over 100 from a probabilistic point of view. Based on this principle, one way to discriminate lncRNAs from mRNAs is by ORF length. For example, the FANTOM project used a maximum ORF length cutoff of 100 codons to differentiate noncoding RNAs from mRNAs [72]. However, some lncRNAs are known to have ORFs longer than 100 codons, while some protein coding genes have fewer than 100 amino acids, such as RCI2A gene in Arabidopsis which encodes a protein of 54 amino acids 


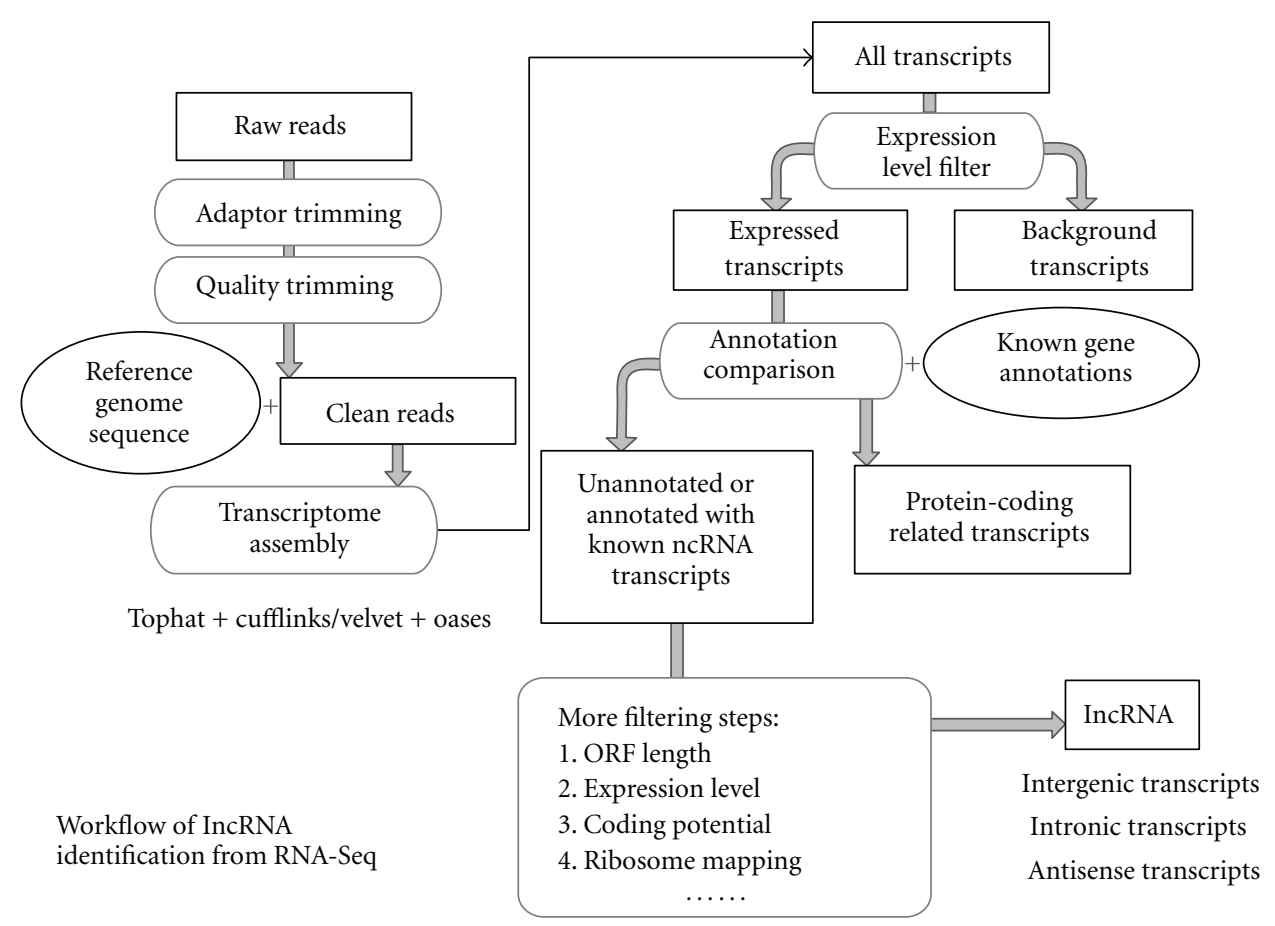

FIgURE 1: Workflow of lncRNA identification from RNA-Seq.

[73]. Thus, this approach may cause misclassification. To overcome the drawbacks of methods based on ORF length, Jia et al. utilize a comparative genomics method to refine ncRNA candidates. They defined the RNA sequences as ncRNAs only if the cDNAs have no homologous proteins longer than 30 amino acids across the mammalian genomes [7]. However, this method relies largely on the completeness of the databases. Therefore, deficiency in protein coding annotation may cause misclassification of lncRNAs as well.

Sequence and Secondary Structure Conservation Strategy. Compared to protein coding genes, noncoding genes are generally less conservative, meaning they are more inclined to mutate [21, 67]. Thus, measuring the coding potential is considered a way of identifying lncRNAs. Codon Substitution Frequency (CSF) is one of the criteria. For example, Guttman et al. used the maximum CSF score to assess the coding potential of a RNA sequence [71]. Clamp et al. and Lin et al. further combined CSF with reading frame conservation (RFC) to discriminate lncRNAs from mRNAs $[74,75]$. Other similar methods include PhyloCSF use a phylogenetic framework to build two phylogenetic codon models that can distinguish coding from noncoding regions [76]. RNAcode combines amino acid substitution with gap patterns to assess the coding potential [77]. There are also methods that explore the conservation of RNA secondary structures to identify lncRNAs, including programs QRNA [78], RNAz [79], and EvoFOLD [80]. However, this approach is limited by lack of common conserved secondary structures specific for lncRNAs.

Machine Learning Strategies. Owing to the complex identities of lncRNAs, recently an increasing number of machine learning-based methods have been developed to integrate various sources of data to distinguish $\operatorname{lncRNAs}$ from mRNAs. Table 1 summarizes the machine learning methods and the features used to train the model for identifying lncRNAs. For instance, CONC utilizes a series of protein features such as amino acid composition, secondary structure, and peptide length, to train a SVM model that distinguishes lncRNAs from mRNAs [18]. CPC (Coding Potential Calculator) also uses SVM for modeling and extracting sequence features and the comparative genomics features to assess the coding potential of transcripts $[19,20]$. Lu et al. developed a machine learning method that integrates GC content, DNA conservation, and expression information to predict lncRNAs in C. elegans [21].

Although the above-described methods have shown their effectiveness in identifying lncRNAs, exceptional cases still remain. For instance, whether an RNA transcript is translated or not may be changeable during the course of evolution. As an example, Xist, a well-known lncRNA, evolves from a protein-coding gene [81]. Besides, some genes are bifunctional, and both the coding and noncoding isoforms exist. The steroid receptor RNA activator (SRA) was characterized as a noncoding RNA previously but the coding product was detected later [82]. Such ambiguity will be clarified when more about lncRNAs are known.

\section{IncRNA Function}

lncRNAs have once been thought as the "dark matter" of the genome, because of our limited knowledge about their functions [83]. With more studies about lncRNAs conducted, it has become clear that lncRNAs have many specific functional features, and are likely to be involved in many diverse 
TABLE 1: Machine-learning methods for identifying lncRNAs.

\begin{tabular}{|c|c|c|c|}
\hline Method & Features & Algorithm & References \\
\hline \multirow{8}{*}{ CONC } & Peptide length & \multirow{8}{*}{ SVM } & \multirow{8}{*}[18]{} \\
\hline & Amino acid composition & & \\
\hline & Hydrophobicity & & \\
\hline & Secondary structure content & & \\
\hline & Percentage of residues exposed to solvent & & \\
\hline & Sequence compositional entropy & & \\
\hline & Number of homologs obtained by PSI-BLAST & & \\
\hline & Alignment entropy & & \\
\hline \multirow{4}{*}{$\mathrm{CPC}$} & ORF prediction quality & & \multirow{4}{*}[19,20]{} \\
\hline & Number of homologs obtained by BLASTX & SVM & \\
\hline & Alignment quality & & \\
\hline & Segment distribution & & \\
\hline \multirow{10}{*}{ Lu et al. } & RNA-seq experiments & & \multirow{10}{*}[21]{} \\
\hline & Tilling arrays & & \\
\hline & poly-A + RNA-seq experiments & Naïve Bayes & \\
\hline & & Bayes Net & \\
\hline & poly-A + tilling arrays & Decision Tree & \\
\hline & GC content & Random Forest & \\
\hline & DNA conservation & Logistic Regression & \\
\hline & Predicted protein sequence conservation & SVM & \\
\hline & Predicted secondary structure free energy & & \\
\hline & Predicted secondary structure conservation & & \\
\hline
\end{tabular}

biological processes in cells. Rather than "dark matter," they may act as necessary functional parts in the genome. These functional features include but are not limited to (i) lncRNAs have conserved splice junctions and introns [84]; (ii) the expression patterns of lncRNAs are tissue- and cell-specific $[12,67]$; (iii) the altered expression of lncRNAs can be found in neurodegeneration, cancer, and other diseases $[9,10]$; (iv) lncRNAs are associated with particular chromatin signatures that are indicative of actively transcribed genes [11, 85]. Below, we will briefly summarize the cellular functions of lncRNAs and molecular mechanisms of their functions.

4.1. Cellular Functions of $\ln c R N A$. With thousands of lncRNAs identified in mammals and other vertebrates [16], a few lncRNAs have been extensively studied, which have shed light on their possible functions. Firstly, lncRNAs are involved in various epigenetic regulations through recruitment of chromatin remodeling complexes to specific genomic loci, such as Xist, Air, and Kcnq1ot1 [22, 43]. Secondly, lncRNAs can regulate gene expression by interacting with protein partners in biological processes like protein synthesis, imprinting (Kcnq1ot1, Air), cell cycle control (TERRA), alternative splicing (MALAT1), and chromatin structure regulation (DNMT3b, PANDA) [9, 10, 38, 71, 85-89]. Thirdly, lncRNAs are involved in enhancer-regulating gene activation (eRNAs), in which cases they may interact directly with distal genomic regions [90]. Fourthly, some lncRNAs serve as interacting partners or precursors for short regulatory ncRNAs [91]. For example, microRNAs (miRNAs) can be generated through sequential cleavage of lncRNAs, while Piwi-interacting RNAs (piRNAs) can be produced by processing a single lncRNA transcript [88].

Recent studies have shown the expression of lncRNA is tissue specific. Loewer et al. studied the expression of lncRNA in global remodeling of the epigenome and during reprogramming of somatic cells to induce pluripotent stem cells (iPSCs). They found some lncRNAs have cell-type specific expression pattern $[26,92]$. Loss-of-function studies on most intergenic lncRNAs expressed in mouse embryonic stem (ES) cells revealed that knockdown of intergenic lncRNAs has major consequences on gene expression patterns, which are comparable to the effects of knockdown of well-known ES cell regulators [93]. This indicated that lncRNAs might play important roles in regulating developmental process. The ENCODE project analyzed the tissue-specific expression of lncRNAs in 31 cell types, and found that many lncRNAs have brain-specific expression pattern $[9,12]$. There are increasing lines of evidences that link dysregulations of lncRNAs to diverse human diseases ranging from neuron diseases to cancer $[9,10]$, suggesting that the involvement of lncRNAs in human diseases can be far more prevalent than previously thought [94]. 
4.2. Molecular Mechanisms of IncRNA. The precise mechanism of how lncRNAs function still remains largely unknown. Currently, there are several hypothesis about it, including (1) RNA:DNA:DNA triplex (trans-); (2) RNA:DNA hybrid; (3) RNA:RNA hybrid of lncRNA with a nascent transcript; (4) RNA-protein interaction (cis-/trans-). Although only (1), (2), and (4) have been experimentally demonstrated so far [14], it is generally thought that lncRNAs may function through the interaction with its partners, such as DNA, RNA, or protein, and serve the following roles: signal, decoy, scaffold, and guide $[11,14]$. Table 2 lists lncRNAs that use different mechanisms when carrying out their functions. Below, we give examples for the above-mentioned mechanisms.

Signal. Some lncRNAs have been reported to respond to diverse stimuli, hinting they may act as molecular signals $[12,24,25,27,35]$. For example, lncRNAs can act as markers for imprinting (Air and Kcnq1ot1), X inactivation (Xist), and silencing (COOLAIR). ChIP-Seq studies showed that the gene-activating enhancers produce lncRNA transcripts (eRNAs) [29, 95], and their expression level positively correlates with that of nearby genes, indicating a possible role in regulating mRNA synthesis. This is supported by a recent Loss-of-Function study that found the knockdown of 7 out of $12 \operatorname{lncRNAs}$ affects expression of their cognate neighboring genes [8].

Decoy. IncRNA can function as molecular decoy to negatively regulate an effector. Gas5 contains a hairpin sequence motif that resembles the DNA-binding site of the glucocorticoid receptor [31]. It can serve as a decoy to release the receptor from DNA to prevent transcription of metabolic genes [14]. Another example is the telomeric repeat-containing RNA (TERRA). It interacts with the telomerase protein through a repeat sequence complementary to the template sequence of telomerase RNA [11,34].

Guide. Upon interaction with the target molecular, IncRNA may have the ability to guide it into the proper position either in cis (on neighboring genes) or in trans (on distantly located genes). The newly found eRNAs appear to exert their effects in cis by binding to specific enhancers and actively engaged in regulating mRNA synthesis $[11,29]$. HOTAIR and HOTTIP are transcribed within the human $H O X$ clusters, and serve as signals of anatomic positions by expressing in cells that have distal and posterior positional identities; they both require the interacting partners to be properly localized to the site of action [6]. In this process, chromosomal looping of the $5^{\prime}$ end of HOXA brings HOTTIP into the spatial proximity of multiple HOXA genes, enforcing the maintenance of H3K4me3 and gene activation [14]. This long-range gene activation mechanism suggests that chromosome looping plays a central role in delivering lncRNA to its site of action $[11,45]$.

Scaffold. Recent studies found that several lncRNAs have the capacity to bind more than two protein partners, where the lncRNAs serve as adaptors to form the functional protein complexes. The telomerase RNA TERC (TERRA) is a classic example of RNA scaffold, and is essential for telomerase function. HOTAIR binds the polycomb complex PRC2 to exert its "signal" function. A recent study found that the $3,700 \mathrm{nt}$ of HOTAIR also interact with a second complex consisting of LSD1, CoREST, and REST to antagonize gene activation, further emphasizing its important role as the scaffold of the functional complex $[11,51]$.

Cis- and Trans-Action of IncRNAs. IncRNAs can be classified as cis- or trans-regulators depending on whether it exerts its function on a neighboring gene on the same allele from which it is transcribed [96]. It was considered that many lncRNAs act as cis-regulators, as the expression of lncRNA is significantly correlated with their neighboring protein-coding genes [97, 98]. However, recent studies have questioned that the positive correlation between $\operatorname{lncRNAs}$ and their neighboring genes may be due to shared upstream regulation (such as, lincRNA-p21 [24] and lincRNA-Sox2 [6]), positional correlation (such as, HOTAIR [6]), transcriptional "ripple effects" [98], and indirect regulation of neighboring genes, instead of the effects of cis-regulation. This was supported by the fact that knock down of different number of lncRNAs had little effect on the expression of neighboring genes [96]. In general, it has been accepted that some lncRNAs are cis-regulators [99, 100], while the vast majority may function as trans-regulators $[6,11,93]$. Recently, some cis-regulating lncRNAs were found to have the capacity to act in trans $[33,101,102]$, highlighting the complexity of lncRNAs.

Although substantial research progresses have been made since the discovery of lncRNAs, it still remains a challenge to understand the functions of lncRNAs. One reason is, unlike protein-coding genes whose mutations may result in severely obvious phenotypes, mutations in lncRNAs often do not cause significant phenotypes [85]. It is likely that lncRNAs may function at specific stage of development process or under specific conditions, and thus condition-specific studies of lncRNAs' phenotypes may be necessary. With more omics data about lncRNAs accumulating, computational prediction of the function of lncRNAs can help to design experiments to accelerate the understanding of lncRNAs.

\section{IncRNA Database}

The current lncRNA databases are summarized in Table 3. lncRNAdb is an integrated database specific for lncRNAs, including annotation, sequence, structural, species, and function categories of lncRNAs [55]. NONCODE is a database about ncRNAs that have been experimentally confirmed. It covers almost all published 73,272 lncRNAs in human and mouse; it also includes expression profiles of $\operatorname{lncRNAs}$ and their potential functions predicted from Coding-Noncoding coexpression network (see below) [56]. LNCipedia is another integrated lncRNA database, which includes 21,488 annotated human lncRNAs. It contains lncRNAs information about the coding potential, secondary structure, and microRNA binding sites [57]. fRNAdb and NRED are databases for ncRNAs including lncRNAs $[58,59]$. 


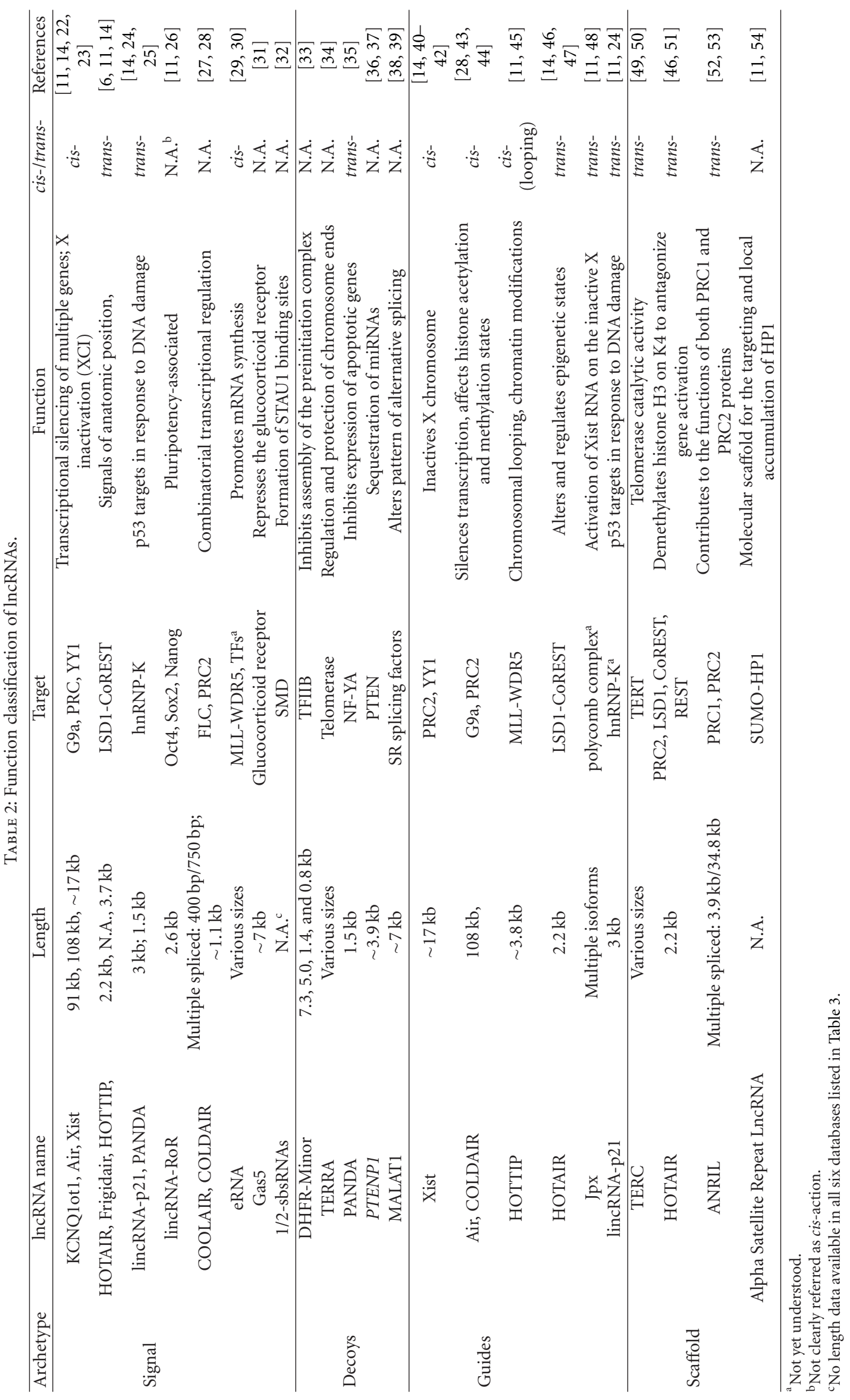


TABLE 3: List of lncRNA databases.

\begin{tabular}{|c|c|c|c|}
\hline Tools & Source & Description & Reference \\
\hline $\operatorname{lncRNAdb}$ & http://www.lncrnadb.org/ & $\begin{array}{l}\text { Contain comprehensive list of lncRNAs } \\
\text { in eukaryotes, and mRNAs with } \\
\text { regulatory roles }\end{array}$ & {$[55]$} \\
\hline NONCODE & http://noncode.org/ & $\begin{array}{l}\text { Integrative annotation of noncoding } \\
\text { RNA }(73,372 \text { lncRNAs) }\end{array}$ & {$[56]$} \\
\hline LNCipedia & http://www.lncipedia.org/ & $\begin{array}{l}21488 \text { annotated human lncRNA } \\
\text { transcripts with secondary structure } \\
\text { information, protein coding potential, } \\
\text { and microRNA binding sites }\end{array}$ & {$[57]$} \\
\hline fRNAdb & http://www.ncrna.org/frnadb/ & $\begin{array}{l}\text { A large collection of noncoding } \\
\text { transcripts including } \\
\text { annotated/unannotated sequences from } \\
\text { H-inv database, NONCODE, and RNAdb }\end{array}$ & {$[58]$} \\
\hline NRED & http://jsm-research.imb.uq.edu.au/nred/cgi-bin/ncrnadb.pl/ & Noncoding RNA Expression Database & [59] \\
\hline
\end{tabular}

The above databases provide great convenience for further analysis and applications of lncRNAs.

\section{Function Prediction of lncRNA}

Computational prediction of lncRNA functions is still at its early development stage. Unlike protein-coding genes whose sequence motifs are indicative of their function, lncRNA sequences are usually not conserved and do not contain conserved sequence motifs [103, 104]. The secondary structures of lncRNA are also not conserved [105]. Thus, it is difficult to infer the function of lncRNAs based on their sequences or secondary structures alone. Since current knowledge suggests that lncRNAs function by regulating or interacting with its partner molecular, current methods focus on exploring the relationships between lncRNAs and protein-coding genes or miRNAs. Below, we will describe several current approaches for predicting the functions of lncRNAs.

6.1. Comparative Genomics Approach. Although most lncRNAs are not conserved, there are lncRNAs that are conserved across species, indicating their essential functions. Amit et al. identified 78 lncRNAs transcripts conserved in both human and mouse, and found 70 are either located within or close ( $<1000 \mathrm{nt}$ distance) to a coding gene that is also conserved in the two genomes [106]. They assumed these lncRNAs might have close functional relationships with the nearby coding genes. However, this approach is limited because of the poor conservation of lncRNAs and cannot be applied at genome scale.

6.2. Coexpression with Coding Genes Approach. Many studied lncRNAs play important regulatory roles, and it is likely that lncRNAs regulating a specific biological process may be coexpressed with the genes involved in the same process. Thus, identifying coding genes that are coexpressed with lncRNAs may help to infer the function of lncRNAs. Based on this assumption, Guttman et al. developed a coexpression based method to predict lncRNAs functions at genome scale [71]. For each lncRNA, they ranked coding genes based on their coexpression level with the lncRNAs, and then performed a Gene Set Enrichment Analysis (GSEA) for the top-ranked genes to identify enriched functional terms corresponding to the lncRNAs. Out of 150 lncRNAs subjected for experimental validation, 85 exhibited the predicted functions, proving the effectiveness of using the coexpressed coding genes to infer the function of lncRNAs from their coexpressed coding genes. According to their predictions, lncRNAs participate in a rather wide range of biological processes such as cell proliferation, development, and immune surveillance. Andrea et al. employed a similar approach to predict the function of lncRNAs during zebrafish embryogenesis [67].

Liao et al. furthered the coexpression idea by constructing a coding-noncoding (CNC) gene coexpression network [107]. In contrast to the GSEA method that collects coding genes coexpressed for each lncRNA, the CNC method considers not only the coexpression between lncRNAs and coding genes, but also within lncRNAs group and coding gene group. When predicting the function of lncRNAs, the CNC method employs two different approaches: the hub-based and the network-module-based. In the hub-based approach, functions are assigned to each lncRNA according to the functional enrichment of its neighboring genes. In the networkmodule-based approach, Markov cluster algorithm (MCL) is used to identify coexpressed functional module in the CNC network; then functions of the module are transferred to the lncRNAs inside the module. Liao et al. applied the CNC method to annotate the functions of 340 mouse lncRNAs, and found these lncRNAs function mainly in organ or tissue development, cellular transport, and metabolic processes.

6.3. Interaction with miRNAs and Proteins Approach. Recent analysis found that lncRNAs share a synergism with miRNA in the regulatory network $[108,109]$. It is likely that some lncRNAs function by binding miRNA. Therefore, identifying well-established miRNAs that bind lncRNAs may help to infer the function of lncRNAs. Jeggari et al. developed an algorithm named miRcode that predicts putative microRNA binding sites in lncRNAs using criteria such as seed complementarity and evolutionary conservation [110]. Jalali et al. constructed a genome-wide network of validated RNA mediated interactions, and uncovered previously unknown 
mediatory roles of lncRNA between miRNA and mRNA (Saakshi Jalali, arXiv preprint). Besides the interaction with miRNA, the interaction of lncRNAs with proteins can also be explored to predict their functions. Bellucci et al. developed a method called "catRAPID" that correlates lncRNAs with proteins by evaluating their interaction potential using physicochemical characteristics, including secondary structure, hydrogen bonding, van der Waals, and so forth [111]. However, unlike the coexpression based approach, the above two approaches were successful in only a number of lncRNAs, partly because the mechanism of how lncRNAs interact with miRNAs and proteins still remains unclear.

6.4. Challenges. Computational prediction of lncRNA functions is still at its primary stage. As the sequence and secondary structure of lncRNAs are generally not conserved, function prediction of lncRNAs mainly relies on their relationships with other moleculars, such as protein coding genes, miRNAs, and proteins. However, the molecular mechanism of how lncRNA function by interacting with other molecular remains largely unknown, making it difficult to develop computational methods to precisely predict the functions of lncRNAs. On the other hand, there are currently only a small number of lncRNAs whose functions are well understood, which makes it difficult to validate and optimize computational algorithms for predicting lncRNA functions. Finally, unlike protein-coding genes that have systematic functional annotation systems, there lacks an annotation system for lncRNA functions, making it difficult to evaluate computational algorithms for function prediction. Nevertheless, the success of predicting lncRNAs using the coexpression based approach has shown promises. With more functional genomics data about lncRNAs available in the near future, more powerful and accurate methods will be developed to help decipher the functions of lncRNAs.

\section{Perspectives}

It has been widely accepted that lncRNAs play important functional roles in cell, though the molecular mechanism of how lncRNAs function remains to be unraveled. In this paper, we have described several currently proposed models about the molecular mechanism of lncRNA functions. One commonality about these models is that lncRNAs function through the interaction with other molecular, including DNA, RNA, and proteins. Given the abundance of lncRNAs in genome, it is likely that the interaction between lncRNAs and other moleculars may be specific. This thus raises the possibility of developing novel methods to target certain lncRNA for gene-specific regulation. However, phenotypic studies of lncRNAs suggested that knockdown of many lncRNAs does not result in obvious phenotypes, making it difficult to understand their functions. Computational prediction of IncRNAs can provide hypothesis about the functions of lncRNAs, and help to design experiments to test them under specific conditions. Yet, it remains a significant challenge to develop effective methods to accurately infer the lncRNA functions, owing to the lack of detailed information about the molecular mechanisms of lncRNAs. In order to develop powerful computational methods, more studies about the derivation of lncRNAs, the molecular mechanism of IncRNAs and tissue-specific, or development-specific expression about lncRNAs are necessary.

\section{Acknowledgment}

This work was supported by the National Natural Science Foundation of China (Grant no. 31071113).

\section{References}

[1] P. Carninci, T. Kasukawa, S. Katayama et al., "The transcriptional landscape of the mammalian genome," Science, vol. 309, pp. 1559-1563, 2005.

[2] E. Birney, J. A. Stamatoyannopoulos, A. Dutta et al., "Identification and analysis of functional elements in $1 \%$ of the human genome by the ENCODE pilot project," Nature, vol. 447, pp. 799-816, 2007.

[3] P. Kapranov, J. Cheng, S. Dike et al., "RNA maps reveal new RNA classes and a possible function for pervasive transcription," Science, vol. 316, no. 5830, pp. 1484-1488, 2007.

[4] J. E. Wilusz, S. M. Freier, and D. L. Spector, " 3 ' end processing of a long nuclear-retained noncoding RNA yields a tRNA-like cytoplasmic RNA," Cell, vol. 135, no. 5, pp. 919-932, 2008.

[5] A. C. Seila, J. M. Calabrese, S. S. Levine et al., "Divergent transcription from active promoters," Science, vol. 322, no. 5909, pp. 1849-1851, 2008.

[6] J. L. Rinn, M. Kertesz, J. K. Wang et al., "Functional demarcation of active and silent chromatin domains in human HOX loci by noncoding RNAs," Cell, vol. 129, no. 7, pp. 1311-1323, 2007.

[7] H. Jia, M. Osak, G. K. Bogu, L. W. Stanton, R. Johnson, and L. Lipovich, "Genome-wide computational identification and manual annotation of human long noncoding RNA genes," RNA, vol. 16, no. 8, pp. 1478-1487, 2010.

[8] U. A. Ørom, T. Derrien, M. Beringer et al., "Long noncoding RNAs with enhancer-like function in human cells," Cell, vol. 143, no. 1, pp. 46-58, 2010.

[9] I. A. Qureshi, J. S. Mattick, and M. F. Mehler, "Long noncoding RNAs in nervous system function and disease," Brain Research, vol. 1338, no. C, pp. 20-35, 2010.

[10] O. Wapinski and H. Y. Chang, "Long noncoding RNAs and human disease," Trends in Cell Biology, vol. 21, no. 6, pp. 354$361,2011$.

[11] K. C. Wang and H. Y. Chang, "Molecular mechanisms of long noncoding RNAs," Molecular Cell, vol. 43, pp. 904-914, 2011.

[12] T. Derrien, R. Johnson, G. Bussotti, A. Tanzer, S. Djebali et al., "The GENCODE v7 catalog of human long noncoding RNAs: analysis of their gene structure, evolution, and expression," Genome Research, vol. 22, pp. 1775-1789, 2012.

[13] M. E. Dinger, K. C. Pang, T. R. Mercer, and J. S. Mattick, "Differentiating protein-coding and noncoding RNA: challenges and ambiguities," PLoS Computational Biology, vol. 4, no. 11, Article ID e1000176, 2008.

[14] J. L. Rinn and H. Y. Chang, "Genome regulation by long noncoding RNAs," Annual Review of Biochemistry, vol. 81, pp. 145-166, 2012.

[15] C. P. Ponting, P. L. Oliver, and W. Reik, "Evolution and functions of long noncoding RNAs," Cell, vol. 136, no. 4, pp. 629641, 2009. 
[16] J.-W. Nam and D. P. Bartel, "Long noncoding RNAs in C. elegans," Genome Research, vol. 22, no. 12, pp. 2529-2540, 2012.

[17] M. C. Tsai, R. C. Spitale, and H. Y. Chang, "Long intergenic noncoding RNAs: new links in cancer progression," Cancer Research, vol. 71, no. 1, pp. 3-7, 2011.

[18] J. Liu, J. Gough, and B. Rost, "Distinguishing protein-coding from non-coding RNAs through support vector machines," PLoS genetics, vol. 2, no. 4, article no. e29, 2006.

[19] S. F. Altschul, T. L. Madden, A. A. Schäffer et al., "Gapped BLAST and PSI-BLAST: a new generation of protein database search programs," Nucleic Acids Research, vol. 25, no. 17, pp. 3389-3402, 1997.

[20] L. Kong, Y. Zhang, Z. Q. Ye et al., "CPC: assess the proteincoding potential of transcripts using sequence features and support vector machine," Nucleic Acids Research, vol. 35, pp. W345-W349, 2007.

[21] Z. J. Lu, K. Y. Yip, G. Wang et al., "Prediction and characterization of noncoding RNAs in C. elegans by integrating conservation, secondary structure, and high-throughput sequencing and array data," Genome Research, vol. 21, no. 5, pp. 276-285, 2011.

[22] R. R. Pandey, T. Mondal, F. Mohammad et al., "Kcnq1ot1 antisense noncoding RNA mediates lineage-specific transcriptional silencing through chromatin-level regulation," Molecular Cell, vol. 32, no. 2, pp. 232-246, 2008.

[23] F. Mohammad, T. Mondal, and C. Kanduri, "Epigenetics of imprinted long noncoding RNAs," Epigenetics, vol. 4, no. 5, pp. 277-286, 2009.

[24] M. Huarte, M. Guttman, D. Feldser et al., "A large intergenic noncoding RNA induced by p53 mediates global gene repression in the p53 response," Cell, vol. 142, no. 3, pp. 409$419,2010$.

[25] T. Hung, Y. Wang, M. F. Lin et al., "Extensive and coordinated transcription of noncoding RNAs within cell-cycle promoters," Nature Genetics, vol. 43, no. 7, pp. 621-629, 2011.

[26] S. Loewer, M. N. Cabili, M. Guttman et al., "Large intergenic non-coding RNA-RoR modulates reprogramming of human induced pluripotent stem cells," Nature Genetics, vol. 42, no. 12, pp. 1113-1117, 2010.

[27] S. Swiezewski, F. Liu, A. Magusin, and C. Dean, "Coldinduced silencing by long antisense transcripts of an Arabidopsis Polycomb target," Nature, vol. 462, no. 7274, pp. 799-802, 2009.

[28] J. B. Heo and S. Sung, "Vernalization-mediated epigenetic silencing by a long intronic noncoding RNA," Science, vol. 331, no. 6013, pp. 76-79, 2011.

[29] T. K. Kim, M. Hemberg, J. M. Gray et al., "Widespread transcription at neuronal activity-regulated enhancers," Nature, vol. 465, no. 7295, pp. 182-187, 2010.

[30] D. Wang, I. Garcia-Bassets, C. Benner et al., "Reprogramming transcription by distinct classes of enhancers functionally defined by eRNA," Nature, vol. 474, no. 7351, pp. 390 397, 2011.

[31] T. Kino, D. E. Hurt, T. Ichijo, N. Nader, and G. P. Chrousos, "Noncoding RNA Gas5 is a growth arrest- and starvationassociated repressor of the glucocorticoid receptor," Science Signaling, vol. 3, no. 107, article no. ra8, 2010.

[32] C. Gong and L. E. Maquat, "LncRNAs transactivate STAU1mediated mRNA decay by duplexing with 39 UTRs via Alu eleme," Nature, vol. 470, no. 7333, pp. 284-288, 2011.
[33] I. Martianov, A. Ramadass, A. Serra Barros, N. Chow, and A. Akoulitchev, "Repression of the human dihydrofolate reductase gene by a non-coding interfering transcript," Nature, vol. 445, no. 7128, pp. 666-670, 2007.

[34] S. Redon, P. Reichenbach, and J. Lingner, "The non-coding RNA TERRA is a natural ligand and direct inhibitor of human telomerase," Nucleic Acids Research, vol. 38, no. 17, Article ID gkq296, pp. 5797-5806, 2010.

[35] T. Hung and H. Y. Chang, "Long noncoding RNA in genome regulation: prospects and mechanisms," RNA Biology, vol. 7, no. 5, pp. 582-585, 2010.

[36] L. Poliseno, L. Salmena, J. Zhang, B. Carver, W. J. Haveman, and P. P. Pandolfi, "A coding-independent function of gene and pseudogene mRNAs regulates tumour biology," Nature, vol. 465, no. 7301, pp. 1033-1038, 2010.

[37] M. S. Song, A. Carracedo, L. Salmena et al., "Nuclear PTEN regulates the APC-CDH1 tumor-suppressive complex in a phosphatase-independent manner," Cell, vol. 144, no. 2, pp. 187-199, 2011.

[38] V. Tripathi, J. D. Ellis, Z. Shen et al., "The nuclear-retained noncoding RNA MALAT1 regulates alternative splicing by modulating SR splicing factor phosphorylation," Molecular Cell, vol. 39, no. 6, pp. 925-938, 2010.

[39] D. Bernard, K. V. Prasanth, V. Tripathi et al., "A long nuclearretained non-coding RNA regulates synaptogenesis by modulating gene expression," EMBO Journal, vol. 29, no. 18, pp. 3082-3093, 2010.

[40] K. Plath, S. Mlynarczyk-Evans, D. A. Nusinow, and B. Panning, "Xist RNA and the mechanism of X chromosome inactivation," Annual Review of Genetics, vol. 36, pp. 233-278, 2002.

[41] J. T. Lee, "The X as model for RNA's niche in epigenomic regulation," Cold Spring Harbor Perspectives in Biology, vol. 2, no. 9, Article ID a003749, 2010.

[42] B. K. Sun, A. M. Deaton, and J. T. Lee, "A transient heterochromatic state in Xist preempts $\mathrm{X}$ inactivation choice without RNA stabilization," Molecular Cell, vol. 21, no. 5, pp. 617-628, 2006.

[43] T. Nagano, J. A. Mitchell, L. A. Sanz et al., "The Air noncoding RNA epigenetically silences transcription by targeting G9a to chromatin," Science, vol. 322, no. 5908, pp. 17171720, 2008.

[44] J. Camblong, N. Iglesias, C. Fickentscher, G. Dieppois, and F. Stutz, "Antisense RNA stabilization induces transcriptional gene silencing via histone seacetylation in S. cerevisiae," Cell, vol. 131, no. 4, pp. 706-717, 2007.

[45] K. C. Wang, Y. W. Yang, B. Liu et al., "A long noncoding RNA maintains active chromatin to coordinate homeotic gene expression," Nature, vol. 472, no. 7341, pp. 120-126, 2011.

[46] A. M. Khalil, M. Guttman, M. Huarte et al., "Many human large intergenic noncoding RNAs associate with chromatinmodifying complexes and affect gene expression," Proceedings of the National Academy of Sciences of the United States of America, vol. 106, no. 28, pp. 11667-11672, 2009.

[47] J. Zhao, T. K. Ohsumi, J. T. Kung et al., "Genome-wide identification of polycomb-associated RNAs by RIP-seq," Molecular Cell, vol. 40, no. 6, pp. 939-953, 2010.

[48] D. Tian, S. Sun, and J. T. Lee, "The long noncoding RNA, Jpx, Is a molecular switch for X chromosome inactivation," Cell, vol. 143, no. 3, pp. 390-403, 2010.

[49] K. Collins, "Physiological assembly and activity of human telomerase complexes," Mechanisms of Ageing and Development, vol. 129, no. 1-2, pp. 91-98, 2008. 
[50] D. C. Zappulla and T. R. Cech, "Yeast telomerase RNA: a flexible scaffold for protein subunits," Proceedings of the National Academy of Sciences of the United States of America, vol. 101, no. 27, pp. 10024-10029, 2004.

[51] M. C. Tsai, O. Manor, Y. Wan et al., "Long noncoding RNA as modular scaffold of histone modification complexes," Science, vol. 329, no. 5992, pp. 689-693, 2010.

[52] Y. Kotake, T. Nakagawa, K. Kitagawa et al., "Long non-coding RNA ANRIL is required for the PRC2 recruitment to and silencing of $\mathrm{p} 15^{\mathrm{INK} 4 \mathrm{~B}}$ tumor suppressor gene," Oncogene, vol. 30, no. 16, pp. 1956-1962, 2011.

[53] K. L. Yap, S. Li, A. M. Muñoz-Cabello et al., "Molecular interplay of the noncoding RNA ANRIL and methylated histone H3 lysine 27 by polycomb CBX7 in transcriptional silencing of INK4a," Molecular Cell, vol. 38, no. 5, pp. 662-674, 2010.

[54] C. Maison, D. Bailly, D. Roche et al., "SUMOylation promotes de novo targeting of HPlalpha to pericentric heterochromatin," Nature Genetics, vol. 43, no. 3, pp. 220-227, 2011.

[55] P. P. Amaral, M. B. Clark, D. K. Gascoigne, M. E. Dinger, and J. S. Mattick, "LncRNAdb: a reference database for long xoncoding RNAs," Nucleic Acids Research, vol. 39, no. 1, pp. D146-D151, 2011.

[56] D. Bu, K. Yu, S. Sun, C. Xie, G. Skogerbo et al., "NONCODE v3. 0: integrative annotation of long noncoding RNAs," Nucleic Acids Research, vol. 40, pp. D210-D215, 2012.

[57] P. J. Volders, K. Helsens, X. Wang, B. Menten, L. Martens et al., "LNCipedia: a database for annotated human lncRNA transcript sequences and structures," Nucleic Acids Research. In press.

[58] T. Kin, K. Yamada, G. Terai et al., "fRNAdb: a platform for mining/annotating functional RNA candidates from noncoding RNA sequences," Nucleic Acids Research, vol. 35, no. 1, pp. D145-D148, 2007.

[59] M. E. Dinger, K. C. Pang, T. R. Mercer, M. L. Crowe, S. M. Grimmond, and J. S. Mattick, "NRED: a database of long noncoding RNA expression," Nucleic Acids Research, vol. 37, no. 1, pp. D122-D126, 2009.

[60] S. K. Michelhaugh, L. Lipovich, J. Blythe, H. Jia, G. Kapatos, and M. J. Bannon, "Mining Affymetrix microarray data for long non-coding RNAs: altered expression in the nucleus accumbens of heroin abusers," Journal of Neurochemistry, vol. 116, no. 3, pp. 459-466, 2011.

[61] T. Babak, B. J. Blencowe, and T. R. Hughes, "A systematic search for new mammalian noncoding RNAs indicates little conserved intergenic transcription," BMC Genomics, vol. 6, article no. 14, 2005.

[62] E. A. Gibb, E. A. Vucic, K. S. Enfield, G. L. Stewart, K. M. Lonergan et al., "Human cancer long non-coding RNA transcriptomes," PLoS One, vol. 6, Article ID e25915, 2011.

[63] T. L. Lee, A. Xiao, and O. M. Rennert, "Identification of novel long noncoding RNA transcripts in male germ cells," Methods in Molecular Biology, vol. 825, pp. 105-114, 2012.

[64] M. Furuno, K. C. Pang, N. Ninomiya et al., "Clusters of internally primed transcripts reveal novel long noncoding RNAs," PLoS Genetics, vol. 2, no. 4, article no. e37, 2006.

[65] W. Huang, N. Long, and H. Khatib, "Genome-wide identification and initial characterization of bovine long non-coding RNAs from EST data," Animal Genetics, vol. 43, pp. 674-682, 2012.

[66] T. Li, S. Wang, R. Wu, X. Zhou, D. Zhu et al., "Identification of long non-protein coding RNAs in chicken skeletal muscle using next generation sequencing," Genomics, vol. 99, pp. 292-298, 2012.
[67] A. Pauli, E. Valen, M. F. Lin, M. Garber, N. L. Vastenhouw et al., "Systematic identification of long noncoding RNAs expressed during zebrafish embryogenesis," Genome Research, vol. 22, pp. 577-591, 2012.

[68] J. R. Prensner, M. K. Iyer, O. A. Balbin et al., "Transcriptome sequencing across a prostate cancer cohort identifies PCAT-1, an unannotated lincRNA implicated in disease progression," Nature Biotechnology, vol. 29, no. 8, pp. 742-749, 2011.

[69] J. Zhao, B. K. Sun, J. A. Erwin, J. J. Song, and J. T. Lee, "Polycomb proteins targeted by a short repeat RNA to the mouse X chromosome," Science, vol. 322, no. 5902, pp. 750756, 2008.

[70] P. J. Park, "ChIP-seq: advantages and challenges of a maturing technology," Nature Reviews Genetics, vol. 10, no. 10, pp. 669680, 2009.

[71] M. Guttman, I. Amit, M. Garber et al., "Chromatin signature reveals over a thousand highly conserved large non-coding RNAs in mammals," Nature, vol. 458, no. 7235, pp. 223-227, 2009.

[72] Y. Okazaki, M. Furuno, T. Kasukawa et al., "Analysis of the mouse transcriptome based on functional annotation of 60,770 full-length cDNAs," Nature, vol. 420, no. 6915, pp. 563-573, 2002.

[73] X. Yang, T. J. Tschaplinski, G. B. Hurst et al., "Discovery and annotation of small proteins using genomics, proteomics, and computational approaches," Genome Research, vol. 21, no. 4, pp. 634-641, 2011.

[74] M. F. Lin, J. W. Carlson, M. A. Crosby et al., "Revisiting the protein-coding gene catalog of Drosophila melanogaster using 12 fly genomes," Genome Research, vol. 17, no. 12, pp. 18231836, 2007.

[75] M. Clamp, B. Fry, M. Kamal et al., "Distinguishing proteincoding and noncoding genes in the human genome," Proceedings of the National Academy of Sciences of the United States of America, vol. 104, no. 49, pp. 19428-19433, 2007.

[76] M. F. Lin, I. Jungreis, and M. Kellis, "PhyloCSF: a comparative genomics method to distinguish protein coding and noncoding regions," Bioinformatics, vol. 27, no. 13, Article ID btr209, pp. i275-i282, 2011.

[77] S. Washietl, S. Findeiß, S. A. Müller et al., "RNAcode: robust discrimination of coding and noncoding regions in comparative sequence data," RNA, vol. 17, no. 4, pp. 578-594, 2011.

[78] E. Rivas and S. R. Eddy, "Noncoding RNA gene detection using comparative sequence analysis," BMC Bioinformatics, vol. 2, article no. 8, 2001.

[79] S. Washietl, I. L. Hofacker, and P. F. Stadler, "Fast and reliable prediction of noncoding RNAs," Proceedings of the National Academy of Sciences of the United States of America, vol. 102, no. 7, pp. 2454-2459, 2005.

[80] J. S. Pedersen, G. Bejerano, A. Siepel et al., "Identification and classification of conserved RNA secondary structures in the human genome," PLoS Computational Biology, vol. 2, no. 4, article no. e33, pp. 251-262, 2006.

[81] L. Duret, C. Chureau, S. Samain, J. Weissanbach, and P. Avner, "The Xist RNA gene evolved in eutherians by pseudogenization of a protein-coding gene," Science, vol. 312, no. 5780, pp. 1653-1655, 2006.

[82] S. Chooniedass-Kothari, E. Emberley, M. K. Hamedani et al., "The steroid receptor RNA activator is the first functional RNA encoding a protein," FEBS Letters, vol. 566, no. 1-3, pp. 43-47, 2004.

[83] E. D. Kim and S. Sung, "Long noncoding RNA: unveiling hidden layer of gene regulatory networks," Trends in Plant Science, vol. 17, pp. 16-21, 2012. 
[84] M. Hiller, S. Findeiß, S. Lein et al., "Conserved introns reveal novel transcripts in Drosophila melanogaster," Genome Research, vol. 19, no. 7, pp. 1289-1300, 2009.

[85] J. S. Mattick, "The genetic signatures of noncoding RNAs," PLoS Genetics, vol. 5, no. 4, Article ID e1000459, 2009.

[86] E. Bernstein and C. D. Allis, "RNA meets chromatin," Genes and Development, vol. 19, no. 14, pp. 1635-1655, 2005.

[87] J. Whitehead, G. K. Pandey, and C. Kanduri, "Regulation of the mammalian epigenome by long noncoding RNAs," Biochimica et Biophysica Acta, vol. 1790, no. 9, pp. 936-947, 2009.

[88] J. E. Wilusz, H. Sunwoo, and D. L. Spector, "Long noncoding RNAs: functional surprises from the RNA world," Genes and Development, vol. 23, no. 13, pp. 1494-1504, 2009.

[89] M. Beltran, I. Puig, C. Peña et al., "A natural antisense transcript regulates Zeb2/Sip1 gene expression during Snaill-induced epithelial-mesenchymal transition," Genes and Development, vol. 22, no. 6, pp. 756-769, 2008.

[90] U. A. Ørom and R. Shiekhattar, "Noncoding RNAs and enhancers: complications of a long-distance relationship," Trends in Genetics, vol. 27, pp. 433-439, 2011.

[91] J. S. Mattick and I. V. Makunin, "Small regulatory RNAs in mammals," Human Molecular Genetics, vol. 14, no. 1, pp. R121-R132, 2005.

[92] T. Nagano and P. Fraser, "No-nonsense functions for long noncoding RNAs," Cell, vol. 145, no. 2, pp. 178-181, 2011.

[93] M. Guttman, J. Donaghey, B. W. Carey, M. Garber, J. K. Grenier et al., "lincRNAs act in the circuitry controlling pluripotency and differentiation," Nature, vol. 477, pp. 295-300, 2011.

[94] R. Johnson, “Long non-coding RNAs in Huntington's disease neurodegeneration," Neurobiology of Disease, vol. 46, pp. 245-254, 2012.

[95] F. De Santa, I. Barozzi, F. Mietton et al., "A large fraction of extragenic RNA Pol II transcription sites overlap enhancers," PLoS Biology, vol. 8, no. 5, Article ID e1000384, 2010.

[96] Z. H. Li and T. M. Rana, "Molecular mechanisms of RNAtriggered gene silencing machineries," Accounts of Chemical Research, vol. 45, pp. 1122-1131, 2012.

[97] J. Ponjavic, P. L. Oliver, G. Lunter, and C. P. Ponting, "Genomic and transcriptional co-localization of proteincoding and long non-coding RNA pairs in the developing brain," PLoS Genetics, vol. 5, no. 8, Article ID e1000617, 2009.

[98] M. Ebisuya, T. Yamamoto, M. Nakajima, and E. Nishida, "Ripples from neighbouring transcription," Nature Cell Biology, vol. 10, no. 9, pp. 1106-1113, 2008.

[99] C. J. Brown, A. Ballabio, J. L. Rupert et al., "A gene from the region of the human $\mathrm{X}$ inactivation centre is expressed exclusively from the inactive X chromosome," Nature, vol. 349, no. 6304, pp. 38-44, 1991.

[100] F. Sleutels, R. Zwart, and D. P. Barlow, "The non-coding Air RNA is required for silencing autosomal imprinted genes," Nature, vol. 415, no. 6873, pp. 810-813, 2002.

[101] J. T. Lee, "Lessons from X-chromosome inactivation: long ncRNA as guides and tethers to the epigenome," Genes and Development, vol. 23, no. 16, pp. 1831-1842, 2009.

[102] K. M. Schmitz, C. Mayer, A. Postepska, and I. Grummt, "Interaction of noncoding RNA with the rDNA promoter mediates recruitment of DNMT3b and silencing of rRNA genes," Genes and Development, vol. 24, no. 20, pp. 22642269, 2010.

[103] A. T. Willingham, A. P. Orth, S. Batalov et al., "Molecular biology: a strategy for probing the function of noncoding RNAs finds a repressor of NFAT," Science, vol. 309, no. 5740, pp. 1570-1573, 2005.

[104] T. R. Mercer, M. E. Dinger, and J. S. Mattick, "Long noncoding RNAs: insights into functions," Nature Reviews Genetics, vol. 10, no. 3, pp. 155-159, 2009.

[105] K. C. Pang, M. E. Dinger, T. R. Mercer et al., "Genomewide identification of long noncoding RNAs in CD8 ${ }^{+} \mathrm{T}$ cells," Journal of Immunology, vol. 182, no. 12, pp. 7738-7748, 2009.

[106] A. N. Khachane and P. M. Harrison, "Mining mammalian transcript data for functional long non-coding RNAs," PLoS One, vol. 5, no. 4, Article ID e10316, 2010.

[107] Q. Liao, C. Liu, X. Yuan et al., "Large-scale prediction of long non-coding RNA functions in a coding-non-coding gene coexpression network," Nucleic Acids Research, vol. 39, no. 9, pp. 3864-3878, 2011.

[108] C. Braconi, T. Kogure, N. Valeri et al., "microRNA-29 can regulate expression of the long non-coding RNA gene MEG3 in hepatocellular cancer," Oncogene, vol. 30, pp. 4750-4756, 2011.

[109] M. S. Ebert and P. A. Sharp, "Emerging roles for natural microRNA sponges," Current Biology, vol. 20, no. 19, pp. R858R861, 2010.

[110] A. Jeggari, D. S. Marks, and E. Larsson, "miRcode: a map of putative microRNA target sites in the long non-coding transcriptome," Bioinformatics, vol. 28, pp. 2062-2063, 2012.

[111] M. Bellucci, F. Agostini, M. Masin, and G. G. Tartaglia, "Predicting protein associations with long noncoding RNAs," Nature Methods, vol. 8, no. 6, pp. 444-445, 2011. 

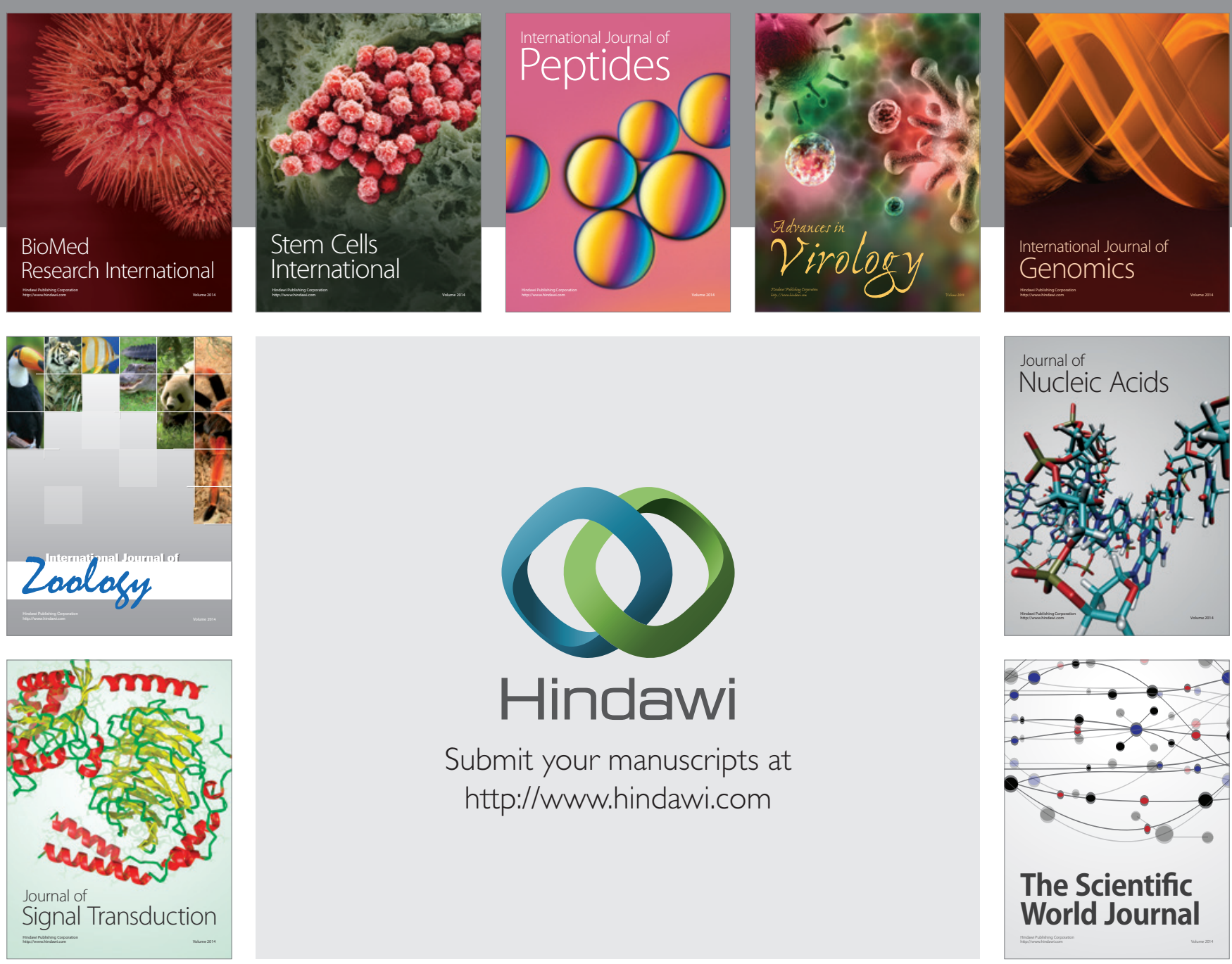

Submit your manuscripts at

http://www.hindawi.com
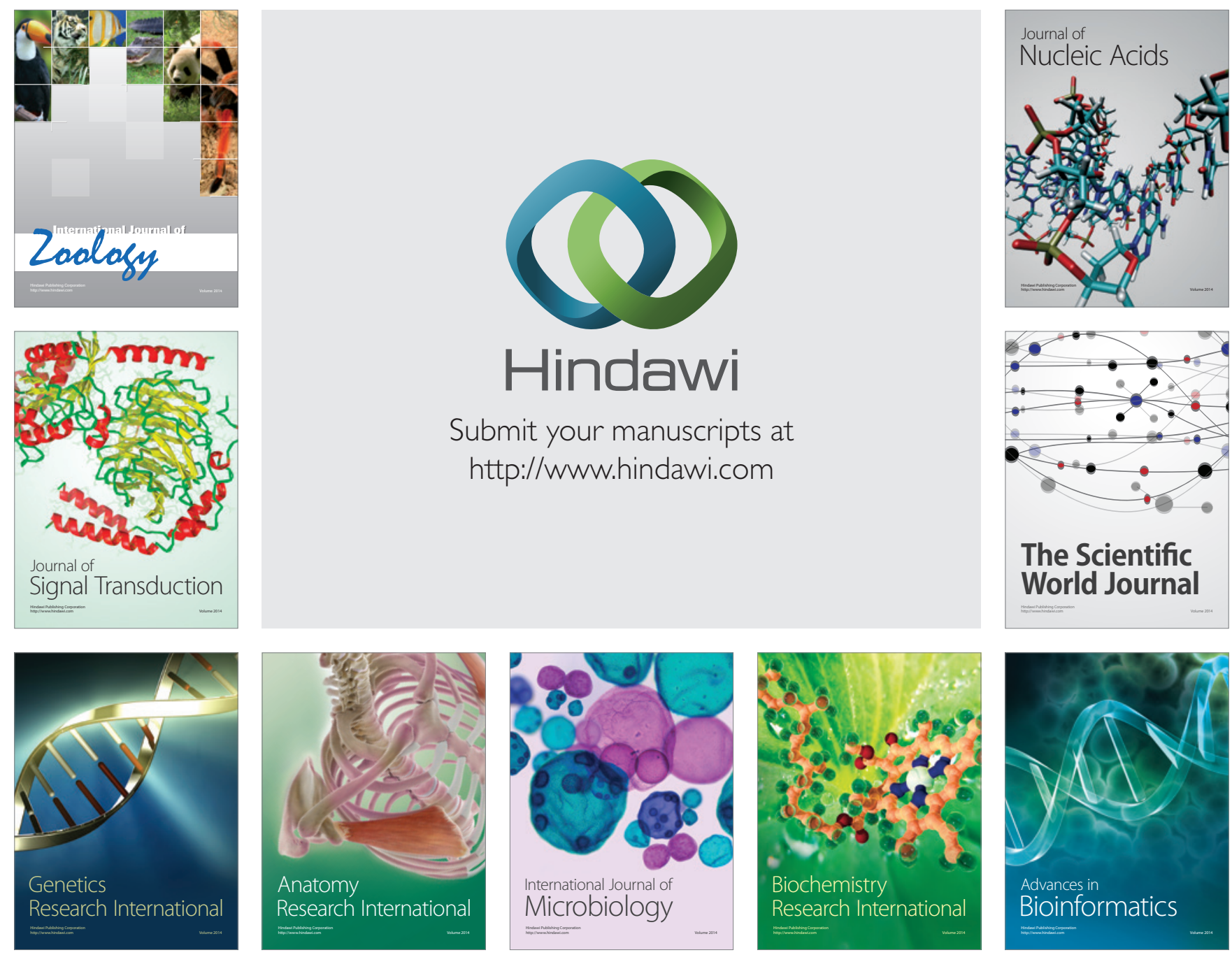

The Scientific World Journal
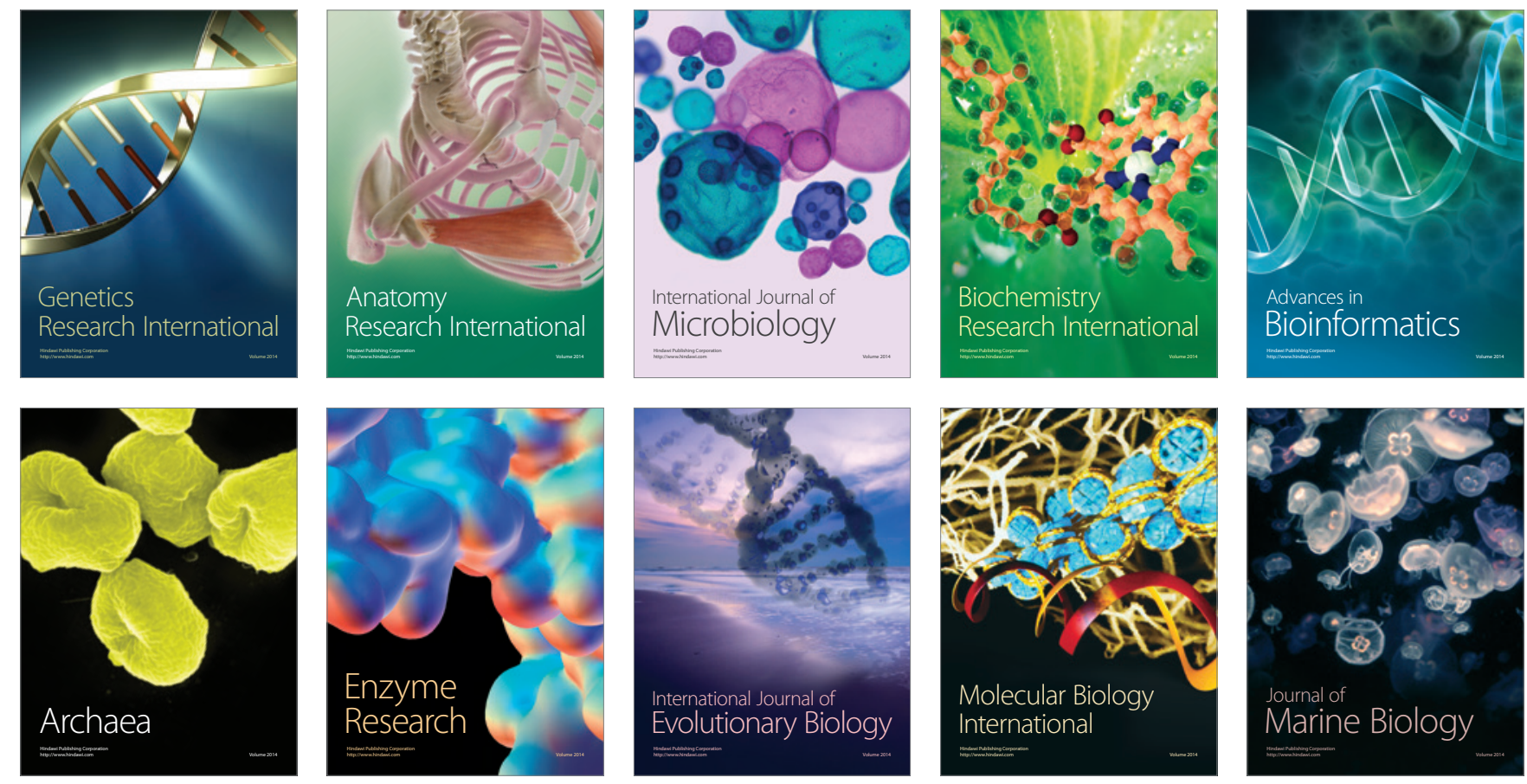